Trauma Berufskrankh 2010 - 12 [Suppl 4]:463-469 DOI 10.1007/s10039-010-1664-1

Online publiziert: 16. September 2010

(c) Springer-Verlag 2010

D. Abitzsch · C. Rusu • H. Lill

Abteilung für Septische und Rekonstruktive Chirurgie,

Klinik für Unfall- und Wiederherstellungschirurgie,

Diakoniekrankenhaus Friederikenstift gGmbH Hannover

\title{
Frühinfekt nach Osteosynthese
}

Häufigkeit

$\mathrm{Zu}$ einem Frühinfekt kommt es

- nach Elektiveingriffen in 1-1,7\%,

- nach geschlossenen Frakturen in 1-5\% und

- nach offenen Frakturen in 2,7-43\% der Fälle.

Die Gesamthäufigkeit der akuten postoperativen und der chronischen Osteitis beträgt

- nach geschlossenen Frakturen 0,5$3 \%$,

- nach offenen Frakturen 2,6-10\% und

- nach Osteosynthesen o,6-3,4\% [5, 8, $9,10,17]$.

\section{Keimspektrum}

Den typischen Erreger einer Osteitis gibt es nicht. Wenngleich bei den aeroben Keimen die verschiedenen Staphylokokkenstämme klar im Vordergrund stehen, können prinzipiell alle Eitererreger eine Osteitis verursachen (• Tab. 1). Gefolgt werden sie in den meisten Statistiken von Pseudomonas aeruginosa, Proteus, Streptokokken, Escherichia coli (E. coli) und Mischinfektion. Bei den anaeroben Keimen spielt Clostridium perfringens klinisch die bedeutendste Rolle.

Im jahrzehntelangen Verlauf einer chronischen Osteitis kommt es häufig zum Erregerwechsel. Über den Fistelgang kommuniziert der Osteitisherd mit der Außenwelt. Der ursprüngliche Infektionserreger wird durch Konkurrenten verdrängt. Hinzu kommt der Selektionsdruck durch eine antibiotische Langzeitbehandlung. Schließlich bleibt oft eine Mischinfektion zurück. In einer solchen können Bakterien unterschiedlicher Ansprüche und Resistenzen existieren [10].

\section{Klassifikation}

Die Einteilung der Osteitis - sowohl in der Diagnostik als auch für die Planung und Durchführung ihrer Therapie - erfolgt von uns in enger Anlehnung an tumorchirurgische Prinzipien anhand des von Hofmann [10] eingeführten Begriffs des Stagings für die pathophysiologische Stadieneinteilung (- Tab. 2), die das klinische Bild des Krankheitsverlaufs am besten wiedergibt.

Das Stadium I, die latente Kontamination, läuft klinisch häufig völlig unauffäl-

\begin{tabular}{|c|c|}
\hline \multirow{6}{*}{$\begin{array}{l}\text { Aerobe } \\
\text { Keime }\end{array}$} & Staphylokokken \\
\hline & Pseudomonas aeruginosa \\
\hline & Proteus \\
\hline & Streptokokken \\
\hline & E. coli \\
\hline & Mischinfektionen \\
\hline \multirow{2}{*}{$\begin{array}{l}\text { Anaerobe } \\
\text { Keime }\end{array}$} & Clostridium perfringens \\
\hline & Alle anderen Keime \\
\hline \multirow{2}{*}{$\begin{array}{l}\text { Keim- } \\
\text { wechsel } \\
\text { im Biotop }\end{array}$} & $\begin{array}{l}\text { Fistelgang: Kontakt zur } \\
\text { Außenwelt }\end{array}$ \\
\hline & $\begin{array}{l}\text { Konkurrenz und Selektions- } \\
\text { druck durch Antibiotikatherapie }\end{array}$ \\
\hline
\end{tabular}

Tab. 2 Pathophysiologische Stadieneinteilung der Osteitis. (Nach [10])

\begin{tabular}{ll} 
Stadium & $\begin{array}{l}\text { Pathophysiologie } \\
\text { I }\end{array}$ \\
$\begin{array}{ll}\text { (stente Kontamination } \\
\text { (I }\end{array}$ & Akute lokale Infektion \\
III & Generalisierte Bakteriämie \\
\hline IV & Septische Metastasierung \\
\hline V & Sepsis, SIRS \\
SIRS, ,systemic inflammatory response syndrome“
\end{tabular}
vor ungeklärt $[4,9,17]$ : 
Trauma Berufskrankh 2010 · 12[Suppl 4]:

463-469

DOI 10.1007/s10039-010-1664-1

๑) Springer-Verlag 2010

D. Abitzsch · C. Rusu $\cdot$ H. Lill Frühinfekt nach Osteosynthese

Zusammenfassung

Die effiziente Therapie der Osteitis ist nur in entsprechend spezialisierten Zentren möglich. Die Behandlung bildet ein einheitliches Ganzes aus Infektsanierung, Weichteildeckung und knöcherner Rekonstruktion. Die Indikation zur Revision und Resektion sollte großzügig gestellt werden. Die Segmentresektion und Kallusdistraktion sind zurzeit die sichersten Methoden zur Sanierung der Osteitis.

\section{Schlüsselwörter}

Osteitis · Knochenerkrankung, infektiöse ·

Rekonstruktion · Resektion · Distraktion

\section{Early infection after osteosynthesis}

\begin{abstract}
Efficient therapy of osteitis is only possible in suitably specialized centers. The treatment consists of an integral whole between infection cleansing, soft tissue covering and bony reconstruction. The indications for revision and resection should be liberally considered. Segment resection and callus distraction are at present the safest methods for cleansing of osteitis.
\end{abstract}

\section{Keywords}

Osteitis · Bone diseases, infectious .

Reconstruction · Resection · Bone lengthening

\section{Tab. 3 Übersicht Labordiagnostik}

\begin{tabular}{llll} 
& Sensitivität & Spezifität & Kosten (EUR) \\
BSG & $90 \%$ & Gering & 3,50 \\
\hline CRP & Hoch & Hoch & $\mathbf{1 1 , 6 0}$ \\
\hline Leukozyten & Gering & Gering & 3,50 \\
\hline Fibrinogen & Gering & Gering & 5,80 \\
$\begin{array}{l}\text { Granulozyten }(P M N) \\
\text { Elastase }\end{array}$ & Hoch & Hoch & 16,90 \\
Neopterin & Gering & Hoch & 32,17 \\
Prokalzitonin & Hoch & Hoch & 50,27 \\
\hline $\begin{array}{l}\text { Zytokine } \\
\text { BSG Blutsenkungsgeschwindigkeit, CRP C-reaktives Protein, PNM polymorphkernig neutrophil }\end{array}$ \\
\hline
\end{tabular}

Tab. 4 Therapiephasen

\begin{tabular}{|ll} 
Phase & Maßnahmen \\
1 & Infektsanierung \\
\hline 2 & Deckung des Weichteildefekts \\
\hline 3 & $\begin{array}{l}\text { Rekonstruktion des Knochen- } \\
\text { defekts }\end{array}$ \\
\hline
\end{tabular}

lig, z. T. ohne pathologische Laborparameter ab. Die Phase II der akuten lokalen Infektion gibt im Wesentlichen das Bild der akuten postoperativen Osteitis wieder. Kommt es zum Übertritt der pathogenen Keime aus dem lokalen Herd in die Blutbahn, entspricht das dem Stadium III, der generalisierten Bakteriämie. In Phase IV bilden sich abgestreute Mikroabszesse in Lungen, Nieren und Gehirn. Zudem kommt es oft zur bakteriellen Mitbesiedlung der Herzklappen. Jede unbeherrschte lokale Infektion kann bei entsprechend ungünstiger Konstellation in die Phase V, die Sepsis, münden [10].

\section{Diagnostik}

Sie stützt sich auf die Anamnese, den klinischen Befund, die Labordiagnostik, die bildgebende Diagnostik mit konventionellem Röntgen, CT (Computertomographie), MRT (Magnetresonanztomographie), 3-Phasen-Szintigraphie und PET/ CT (PET: Positronenemissionstomographie) sowie die Bakteriologie.

\section{Anamnese und klinischer Befund}

Die Anamnese weist in den meisten Fällen den Weg. Verzögerte Wundheilung und Beschwerdepersistenz mit Sekretion und erhöhten Temperaturen sind untrügerische Hinweise auf eine Infektion.
Die klinische Symptomatik ist oft sehr unspezifisch, klassische Entzündungszeichen fehlen häufig. Wenn sich im Anschluss an ein Trauma oder eine Frakturversorgung die aufgetretenen Entzündungszeichen nicht binnen weniger Tage zurückbilden, ist an eine postoperative Infektion zu denken. Postoperativ erhöhte Temperaturen $\left(<38,5^{\circ} \mathrm{C}\right)$ sollten sich nach 3-4 Tagen normalisieren $[2,8,10,13$, $14,17]$.

\section{Labordiagnostik}

In ihr zeigen sich nur beim Frühinfekt deutlich erhöhte Werte, beim Spätinfekt dagegen liegen die Laborarameter nicht selten im Normbereich, insbesondere wenn eine Fistel für regelmäßigen Sekretabfluss sorgt. Im Rahmen des operativen Sanierungsprogramms ist das CRP (C-reaktives Protein) als ein sehr sensibler und spezifischer Verlaufsparameter anzusehen (- Tab. 3). Die Granulozytenelastase sowie das Prokalzitonin weisen ebenfalls eine hohe Sensibilität sowie Spezifität auf, sind aber deutlich kostenungünstiger nachzuweisen, und die entsprechenden Methoden sind nicht ubiquitär verfügbar. Dahingegen zeigen die Leukozytenzahlen nur eine geringe Sensitivität sowie Spezifität $[2,10,16,17]$.

\section{Bildgebende Diagnostik}

Sonographie. Sie bringt über den klinischen Befund hinaus (Schwellung, Gelenkerguss) nur wenig zusätzliche Informationen. Es lassen sich lediglich Aussagen über die Konsistenz und die Größe von Abszessen und Gelenkergüssen so- 
Abb. 1 Frühinfekt
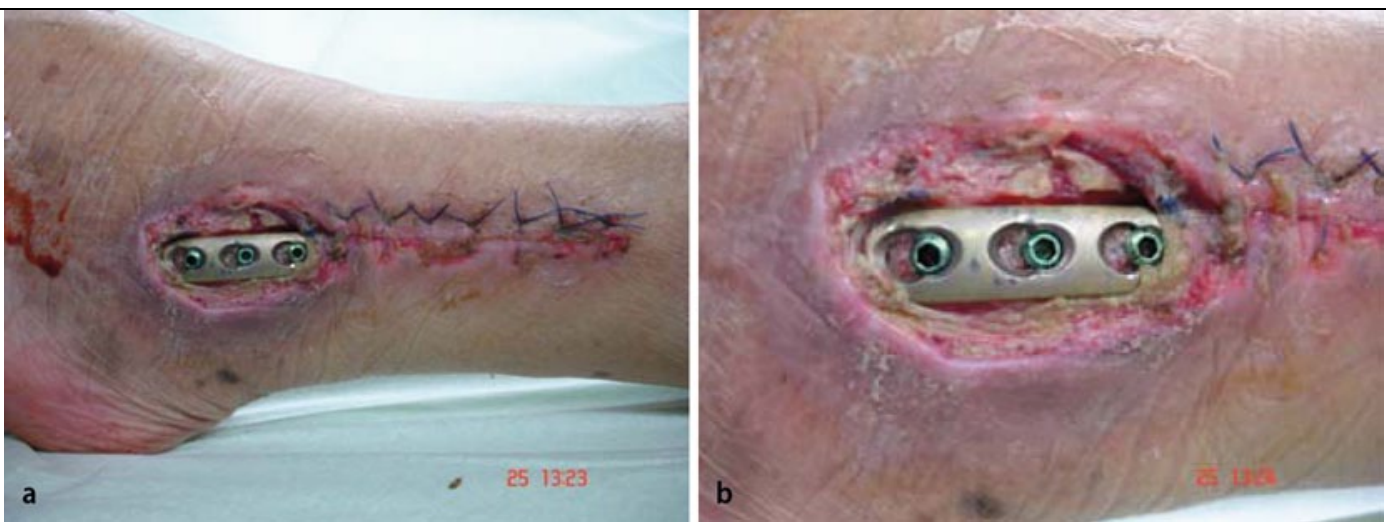

Abb. $2 \triangleright$ Prädisponierende Faktoren, DBS Durchblutungsstörung

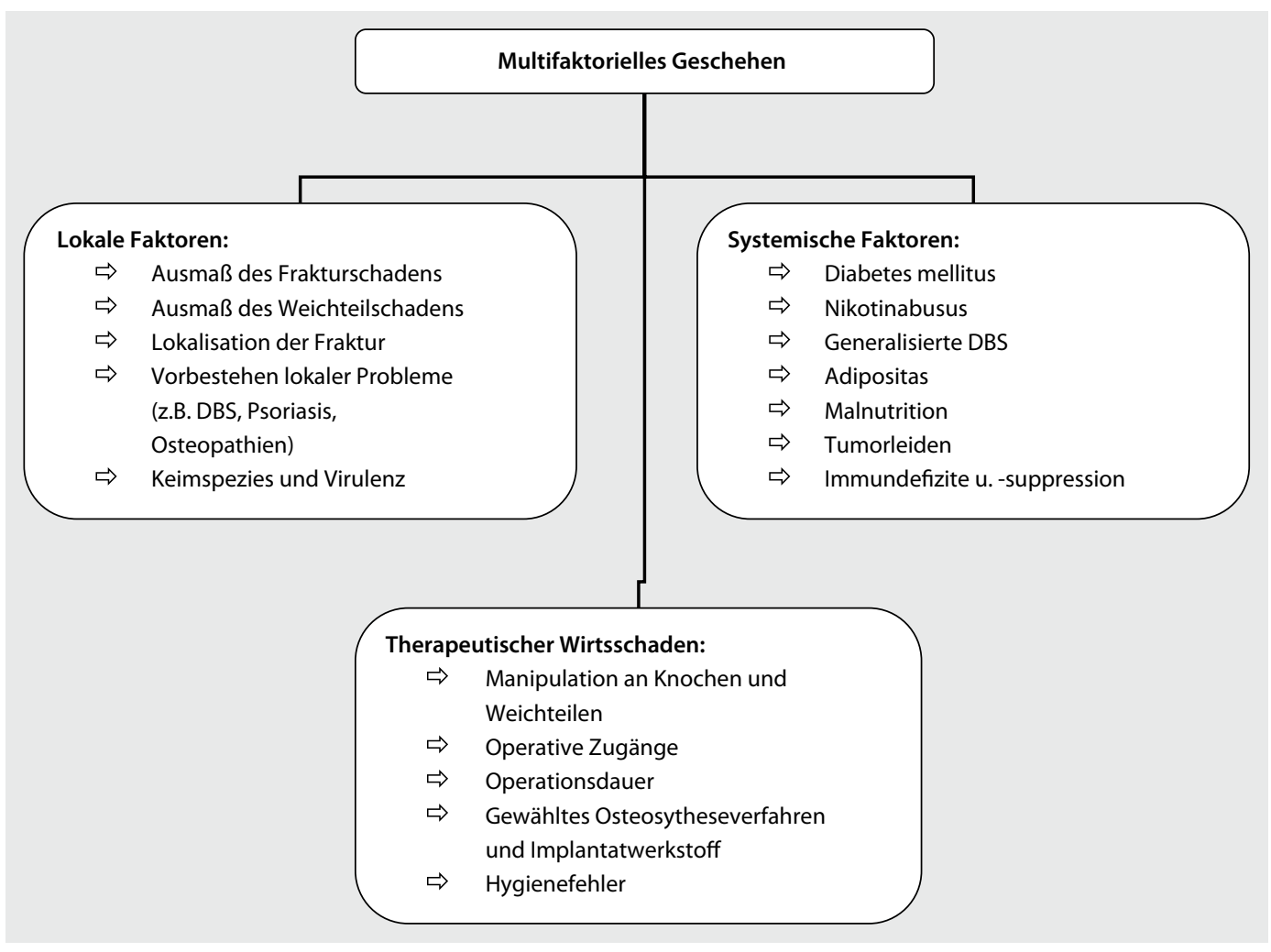

wie zur Dicke der Synovialmembran treffen $[2,10,16,17]$.

Konventionelle Röntgendiagnostik. Sie zeigt beim Frühinfekt keinerlei radiologische Veränderungen. Beim Spätinfekt, frühestens jedoch nach 2-3 Wochen wenn überhaupt - finden sich Sklerosierungen, Osteolysen, Sequestrierungen, periostale Reaktionen und ein Aufbrauch des Gelenkspaltes $[1,2,10,12]$.

Computertomographie. Sie kann insbesondere zum Auffinden kleinerer Knochensequester einen wertvollen Beitrag liefern. Darüber hinaus ermöglicht sie einen Einblick in das Ausmaß der Demineralisation $[1,10,12,16]$.

Abb. 3 Frühinfekt im Magnetresonanztomogramm

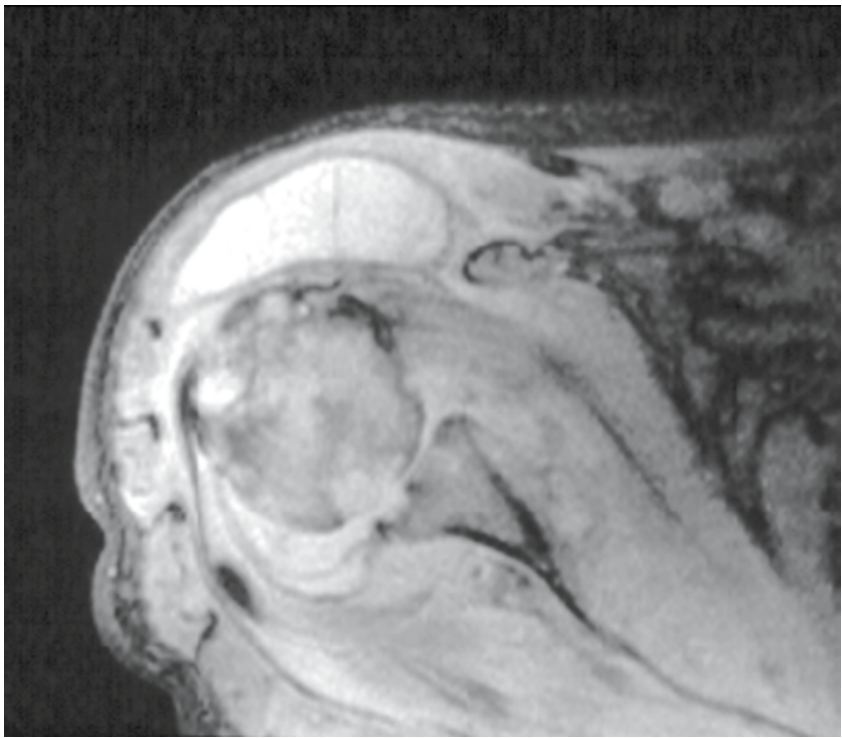




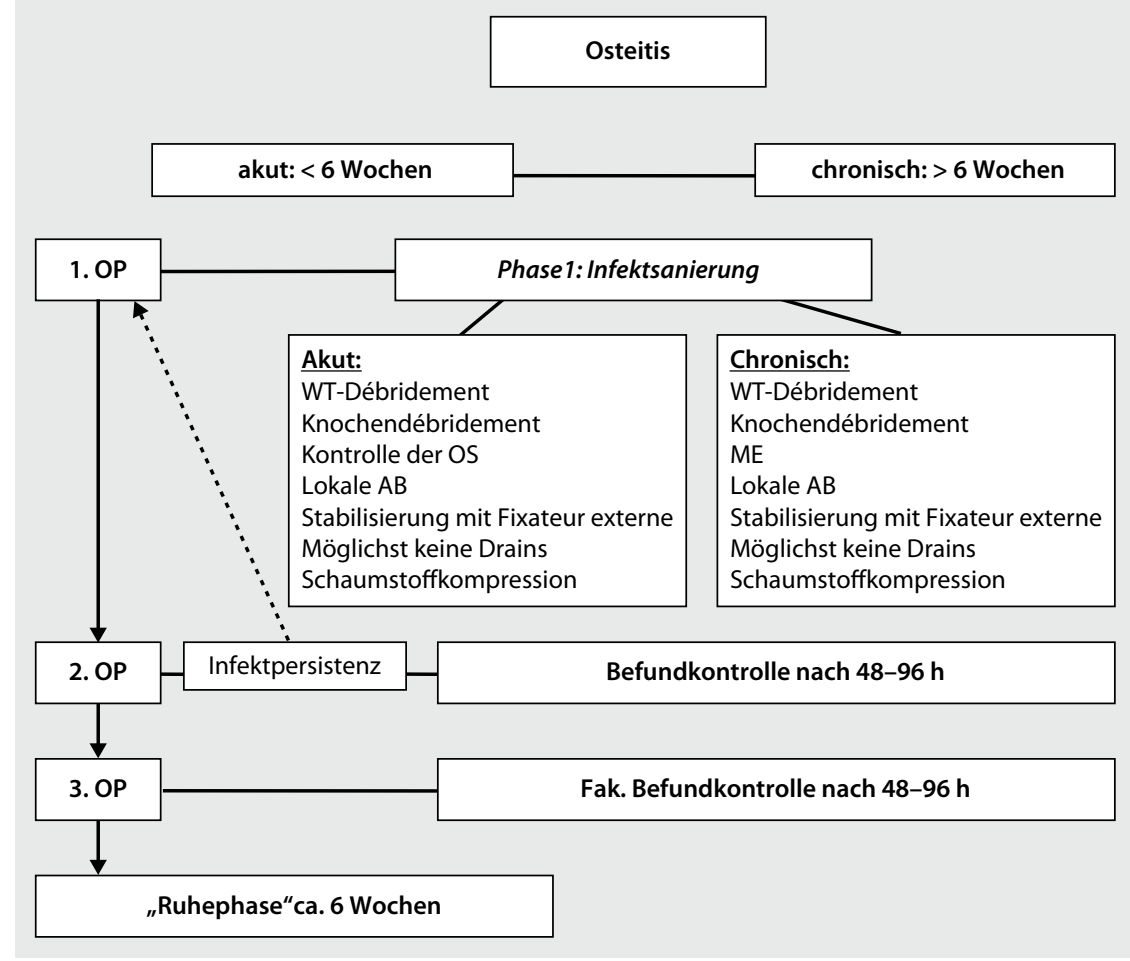

Abb. $4 \Delta$ Programmiertes Sanierungsprogramm, $A B$ Antibiotikatherapie, ME Materialentnahme, $O P$ Operation, OS Osteitis, WTWeichteil. (Mod. nach $[17,18]$ )
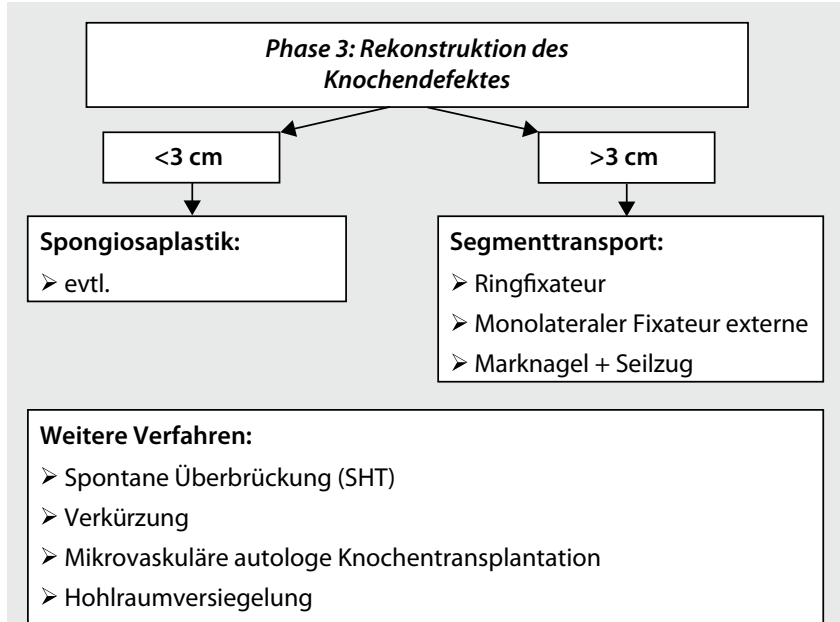

Magnetresonanztomographie. Sie ist Mittel der Wahl beim Frühinfekt ( $\bullet$ Abb. 3) und ermöglicht Aussagen zur Ausdehnung und Lokalisation der Infektionen in Weichteilen und Mark $[10,12,16]$.

3-Phasen-Szintigraphie. Sie weist eine hohe Sensitivität mit $86 \%$ bei einer nur geringen Spezifität von $72-84 \%$ auf. Das bedeutet, dass eine negative Szintigraphie zwar eine Infektion mit nahezu an Sicherheit grenzender Wahrscheinlichkeit ausschließt, andererseits eine positive Szinti-

\section{Bakteriologie}

Sie ist das wichtigste diagnostische Kriterium. Voraussetzung ist die exakte Entnahme der mikrobiologischen Proben, welche unter sterilen Bedingungen zur Vermeidung von Kontamination erfolgen sollte. Es sollten immer mehrere Entnahmestellen in situ mit einem minimalen Probevolumen von etwa $1 \mathrm{~cm}^{3}$ gewählt werden. Wichtig sind ein schneller Transport und eine rasche Weiterverarbeitung. Intraoperativ entnommene Knochen- und Gewebeproben mit kulturellem Nachweis von Mikroorganismen sind der sicherste Beweis einer Infektion, 3 positive Proben mit gleichem Keim und entsprechender Klinik sind beweisend. In 25\% der Proben gelingt kein Erregernachweis [6, 10, 11, 14, $16,17]$.

\section{Therapie}

Ziel der Behandlung sind in hierarchischer Reihenfolge

- die Infekteradikation oder -beruhigung,

- die Schmerzreduktion sowie

- die Erhaltung oder Rekonstruktion belastbarer und funktionsfähiger Extremitäten $[2,17]$.

Die Therapie erfolgt in unserem Haus nach einem genau festgelegten Algorithmus. Unser Revisionskonzept sieht eine verkürzte programmierte Etappenlavage in 2- bis 3-tägigem Abstand vor. Das Vorgehen gliedert sich dabei in 3 Phasen (• Tab. 4, [10]):
Abb. 54 Phase 3: Rekonstruktion des Knochendefekts, SHT Schädel-Hirn-Trauma

\section{Infektsanierung}

In der ersten Phase steht die Infektsanierung im Vordergrund. Planung und Durchführung des Ersteingriffs erfolgen nach streng tumorchirurgischen Grundsätzen (• Abb.4). Bei jedem Eingriff werden mehrere Gewebeproben (nicht nur Abstriche!) aus der Umgebung entnommen.

Der Eingriff beginnt mit einem radikalen Knochen- und Weichteildébridement sowie einer konsequenten Nekrektomie aller avitalen Strukturen. Gegebenenfalls wird das einliegende Implantat gewechselt. Danach wird der Situs mit- 

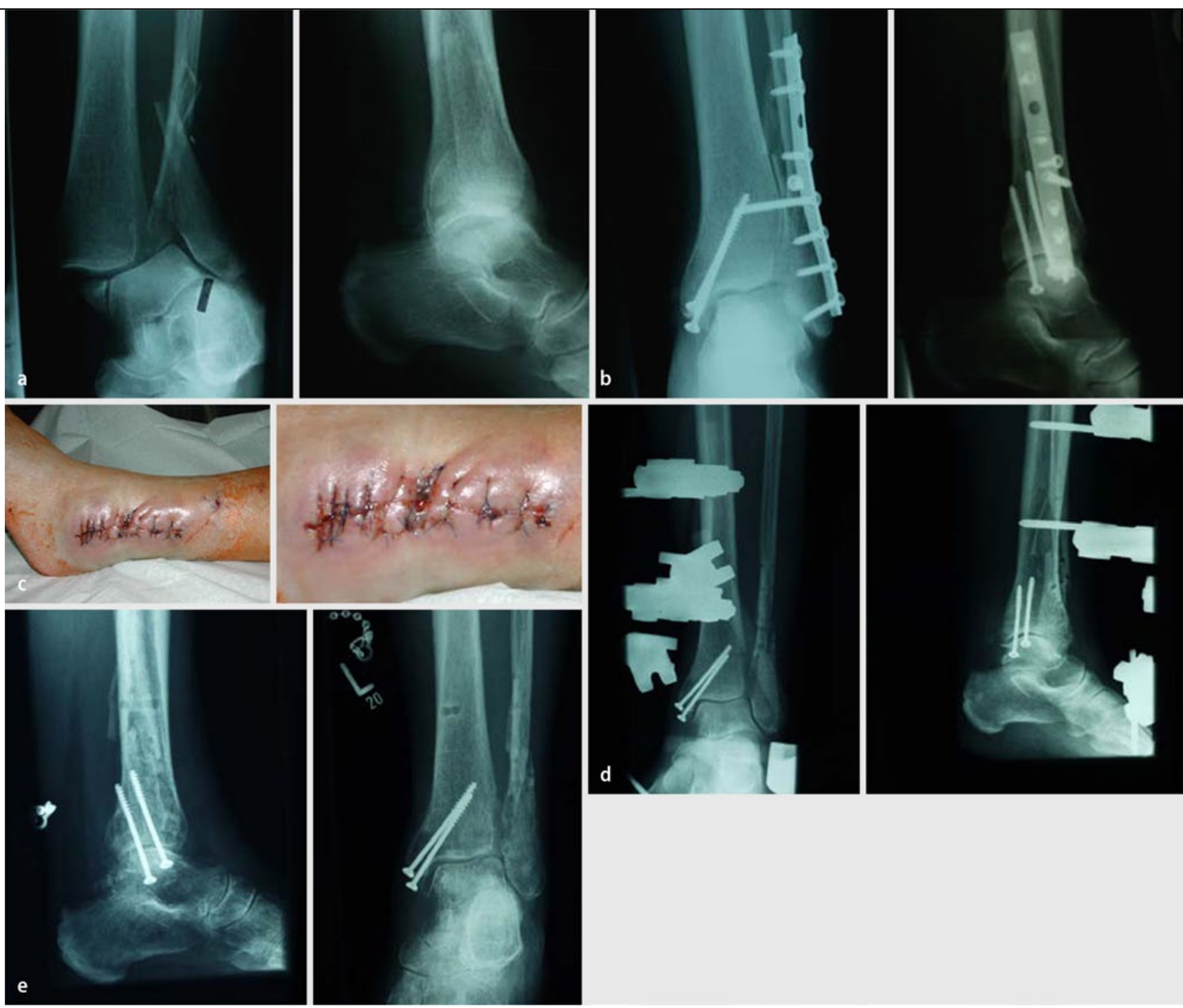

Abb. $6 \Delta$ Fall 1, 73 Jahre, weiblich, bimalleoläre OSG-Luxationsfraktur links, a Unfallbilder, b Versorgung am Unfalltag, c postoperative Infektion im Sinne eines Frühinfektes, $\mathbf{d}$ Zustand nach vollständige Implantatentfernung und Anlage eines tibiometatarsalen Fixateur externe, e Ausheilungsergebnis nach 16 Wochen

tels Jetlavage (3-5 l Ringer-Lösung) hydromechanisch gereinigt und ein lokales Antiseptikum mit einer Einwirkzeit von mindestens 5 min instilliert, wie Octinesept oder verdünnte Betaisodonalösung in Form getränkter Kompressen. Nach deren Entfernen erfolgen das nochmalige sterile Abwaschen und Abdecken des Operationsgebiets sowie der Wechsel von Handschuhen und Instrumentarium. In der nun folgenden sauberen Operationsphase wird ein testgerechtes lokales Antibiotikum, wie Gentacoll oder Septocoll, appliziert. Wichtig ist die schnelle Wirkstofffreisetzung binnen 2-3 Tagen bis zur nächsten Revision. Gelingt der Wundverschluss nicht spannungsfrei oder erscheint die Durchblutung gefährdet, emp- fiehlt sich die Anwendung eines Vakuumverbands $[2,9,10]$.

Zwischenzeitlich muss die Fraktur in einer Gipsschiene, mit einem Fixateur externe oder einem Wechselnagel stabilisiert werden $[5,10,17]$. Wird nicht spätestens nach der 3. Operation Infektfreiheit erzielt, muss das Implantat aufgegeben werden [17]!

\section{Defektdeckung}

Ohne eine qualitativ und quantitativ ausreichende Haut- und Weichteildeckung über dem sanierten knöchernen Areal lässt sich dauerhaft keine rezidivfreie Ausheilung erzielen. Das von uns propagierte, phasengerechte Behandlungskon- zept erfordert eine enge interdisziplinäre Zusammenarbeit zwischen Unfallchirurgie und plastischer/rekonstruktiver Chirurgie. Diese versetzt uns heute in die Lage, im Sinne der Infekteradikation weitaus kompromissloser im Gesunden zu resezieren als dies früher der Fall war. Die notwendige Vorhaltung für mikrovaskuläre Lappentechniken lässt erkennen, dass eine effiziente Behandlung der Osteitis nur in Zentren, die über eine leistungsstarke plastisch-chirurgische Abteilung verfügen, möglich ist [10]. 


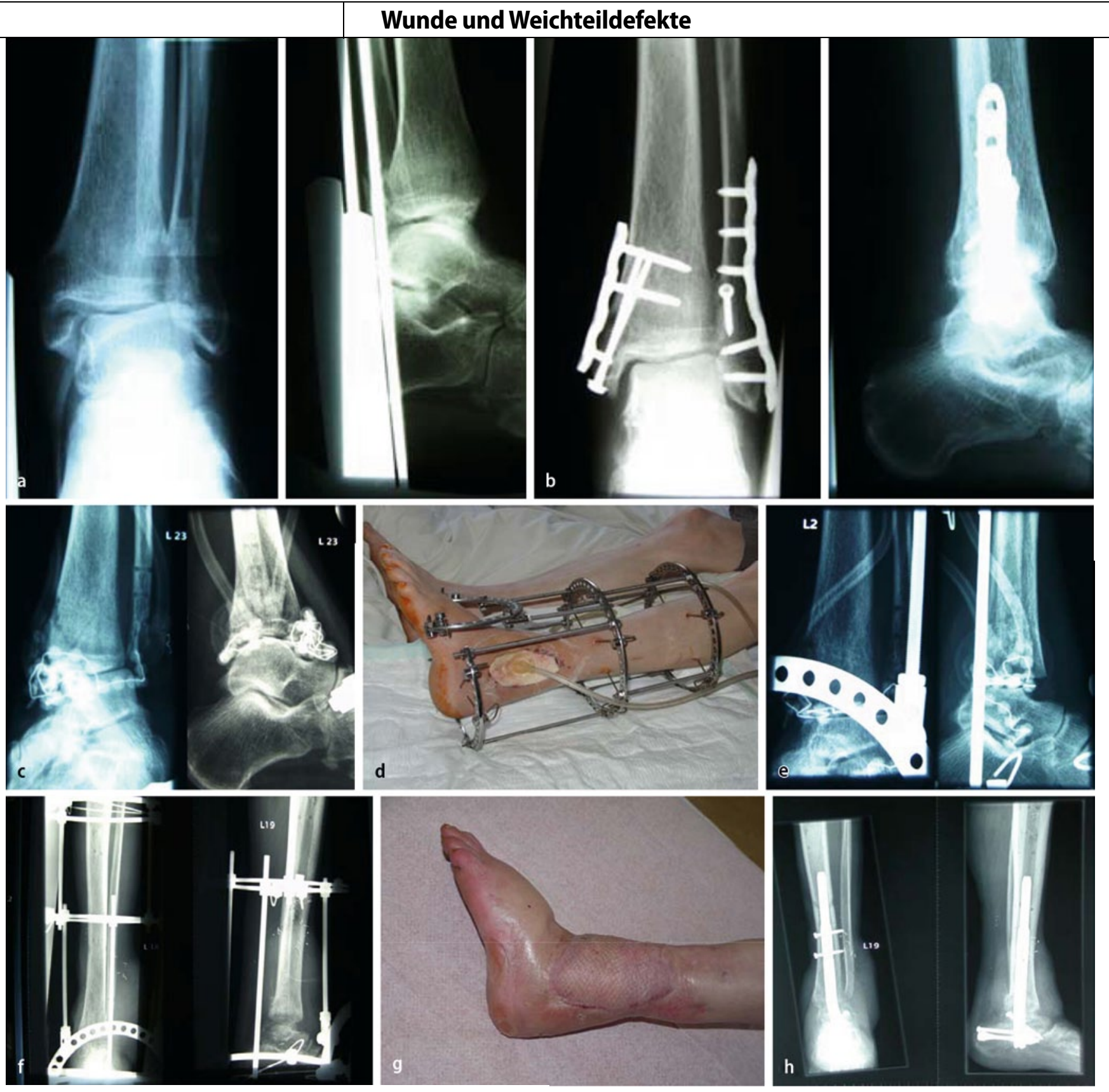

Abb. 7 \ Fallbeispiel 2, 55-jähriger Mann, bimalleoläre OSG-Luxationsfraktur links am 01.05.2008, a Unfallbilder, b Versorgung am gleichen Tag, c 2 Wochen nach dem Trauma vollständige Materialentfernung mit Anlage eines gelenküberbrückenden Fixateur externe, $\mathbf{d}$,e 3 Wochen nach dem Trauma Gelenkflächenresektion mit Anlage eines llisarov-Ringfixateurs im Sinne einer Distraktionsarthrodese, f Verlaufskontrolle bei Zustand nach lappenplastischer Deckung, $\mathbf{g}, \mathbf{h}$ Verlaufskontrolle bei Zustand nach OSG/USG-Arthrodese mittels Rückfußarthrodesennagel

\section{Rekonstruktion der knöchernen Defekte}

Sie erfolgt nach einem infektfreien Intervall von mindesten 6 Wochen (• Abb. 5). Ergibt das anschließend durchgeführte Restaging ein mikrobiologisch negatives Ergebnis, erfolgt der Defektaufbau, bei einer Defektstrecke unter $3 \mathrm{~cm}$ mittels Spongiosaplastik, evtl. ergänzt durch GPS („gravitational platelet separation“), bei Defekten über $3 \mathrm{~cm}$ mittels Segmenttransport durch Kallusdistraktion. Bei kurzstreckigen Defekten kann eine Verkürzung der Extremitäten erwogen werden. Bei Patienten mit schweren Schädel-Hirn-Traumen kann es auch zu einer spontanen Überbrückung kommen. Bei Kindern und Jugendlichen bringen gefäßgestielte autologe Knochentransplantate (Fibula, Rippe, Beckenkamm) erstaunlich gute Resultate.
Ist die mechanische Kontinuität des Knochens nicht durchbrochen, muss ggf. eine definitive Hohlraumversiegelung erfolgen. Diese ist durch autologe Spongiosa oder in Ausnahmefällen durch PMMAKetten (PMMA: Polymethylmethacrylat) möglich $[2,10,16,17,18]$. 


\section{Komplikationen}

Die schwerwiegendste Komplikation ist das Rezidiv. Auch bei vermeintlich erfolgreich sanierten blanden Knochen- und Weichteilverhältnissen muss noch über Jahre mit einer Persistenz der ehemaligen Infektionserreger gerechnet werden. Die Rezidivrate beträgt etwa $15 \%$, in der Literatur bis zu $80 \%$

Weitere Komplikationen sind in Fehlstellung verheilte lange Röhrenknochen, Spontanfrakturen, postinfektiöse Arthrosen der Nachbargelenke, Infektpseudarthrosen, die generalisierte Amyloidose und nicht zuletzt das Fistelkarzinom [10].

\section{Kasuistik}

Fall 1. Die 73-jährige Patientin hatte sich im Rahmen eines Supinationstraumas eine bimalleoläre OSG-Luxationsfraktur (OSG: oberes Sprunggelenk) links zugezogen. Noch am Unfalltag erfolgten die offene Reposition und kombinierte Platten- und Schraubenosteosynthese (D Abb. 6a,b).

Am 10. postoperativen Tag kam es zu einer massiven postoperativen Infektion im Sinne eines Frühinfektes, sodass die Revision des Innenknöchels mit Anlage eines VACVerbandes (VAC: „vacuum assisted closure") erforderlich wurde. Da es bei der 3 Tage später durchgeführten, programmierten Lavage zu keiner deutlichen Befundbesserung kam, erfolgten die vollständige Implantatentfernung und Anlage eines tibiometatarsalen Fixateur externe (- Abb. 6c,d). Nach Infektberuhigung konnte die Wunde 3 Wochen später über dem Innenknöchel mit Spalthaut gedeckt werden.

Nach Entfernung des Fixateur externe mit einer Tragezeit von insgesamt 12 Wochen erfolgte nach Teilbelastung mit $20 \mathrm{~kg}$ für 2 Wochen der schmerzadaptierte Belastungsaufbau (• Abb. 6e).

Fall 2. Der 55-jährige Mann hatte sich im alkoholisierten Zustand beim Fußballspielen eine bimalleoläre OSG-Luxationsfraktur links zugezogen. Diese wurde noch am Unfalltag offen reponiert und mittels kombinierter Platten- und Schraubenosteosynthese versorgt ( $\mathbf{D}$ Abb. 7a,b). Der postoperative Verlauf war aufgrund der Noncompliance - der Patient belas- tete ohne Orthese voll - kompliziert. Zunächst kam es zu einer Wundheilungsstörung und im weiteren Verlauf zu einer akuten postoperativen Osteitis.

Ziel des durchgeführten Revisionsprogramms war anfangs der Erhalt der Osteosynthese. Da jedoch keine rasche Infektfreiheit erzielt werden konnte, erfolgte 2 Wochen nach dem Trauma die vollständige Materialentfernung mit Anlage eines gelenküberbrückenden Fixateur externe. In der Folge kam es dennoch zu einer Mitbeteiligung zunächst des OSG und dann auch des unteren Sprunggelenks (USG), sodass letztlich die Gelenkflächenresektion mit Anlage eines Ilisarov-Ringfixateurs im Sinne einer Distraktionsarthrodese durchgeführt werden musste ( $\mathbf{A b b}$. 7ce). Nachdem letztlich eine Infektberuhigung erzielt werden konnte, erfolgte die Defektdeckung mittels freiem M.-latissimus-dorsi-Lappen. Der weitere Verlauf gestaltete sich kompliziert, es kam zur Lappennekrose und Thoraxwandphlegmone im Bereich der Entnahmestelle, sodass sich die knöcherne Rekonstruktion des Sprunggelenks erheblich verzögerte. Die Osteitis blieb jedoch während des gesamten Zeitraums beruhigt.

Die Rekonstruktion erfolgte mittels Expert-Rückfuß-Arthrodesennagel. Da es sich um ein intramedulläres Verfahren handelt, wird in jedem Fall ein mikrobiologisches Restaging durchgeführt, in diesem Zusammenhang erfolgt gleichzeitig auch das Anfrischen der Gelenkflächen. Bei mikrobiologischer Infektfreiheit wurde 7 Monate nach dem Trauma die OSG/USG-Arthrodese mit dem Expert-Rückfuß-Arthrodesennagel durchgeführt (• Abb. 7f-h).

\section{Korrespondenzadresse}

\section{Dr. D. Abitzsch}

Abteilung für Septische

und Rekonstruktive Chirurgie, Klinik für Unfall-

und Wiederherstellungschirurgie,

Diakoniekrankenhaus

Friederikenstift gGmbH Hannover,

Marienstraße 37, 30171 Hannover

Dirk.Abitzsch@ddh-gruppe.de

Interessenkonflikt. Der korrespondierende Autor gibt an, dass kein Interessenkonflikt besteht.

\section{Literatur}

1. Bohndorf K (1996) Bildgebende Diagnostik der akuten und chronischen Osteomyelitis. Radiologe 36:786-794

2. Bühler M, Engelhardt M, Schmidt H (2003) Septische postoperative Komplikationen. Springer, Berlin Heidelberg New York

3. Carsenti-Etesse H, Doyon F, Desplaces N et al (1999) Epidemology of bacterial infection during management of open leg fractures. Eur J Clin Microbiol Infect Dis 18:315-323

4. Cierny G III, Mader JT, Penninck JJ (1985) A clinical staging system for adult osteomyelitis. Contem Orthop 10:17-37

5. Diefenbeck M, Hofmann GO (2003) Behandlungsstrategien bei Frühinfekt nach operativer Frakturversorgung. Trauma Berufskrankh [Suppl 2] 5: s309-s312

6. Frommelt L, Herrmann M (2004) Klassische mikrobiologische Diagnostik im Skelettbereich und neue Methoden. In: Hendrich C, Frommelt L, Eulert $\mathrm{J}$ (Hrsg) Septische Knochen- und Gelenkchirurgie. Springer, Berlin Heidelberg New York

7. Gentry LO (1988) Osteomyelitis: options for diagnosis and management. J Antimicrob Chemother 21:115-128

8. Hendrich C, Frommelt L, Eulert J (2004) Septische Knochen- und Gelenkchirurgie. Springer, Berlin Heidelberg New York

9. Heppert V, Wentzensen A (2005) Die akute Osteitis. OP J 21:224-230

10. Hofmann GO (2004) Infektionen der Knochen und Gelenke. Urban \& Fischer, München

11. Kujath P, Rodloff AC (2003) Chirurgische Infektionen, aktuelle Aspekte zur Diagnostik und Therapie. UniMed, Bremen

12. Linke R, Weidemann H, Militz M (2009) Bildgebende Diagnostik der Osteitis. Szintigraphie, PET oder MRT? Trauma Berufskrankh [Suppl 2] 11:193202

13. Neugebauer C, Graf R (2004) Expert opinion problems in the evaluation of osteomyelitis. Orthopäde 33:603-611

14. Schmelz A, Kinzl L, Osteitis ET (2006) Infektionen des Bewegungsapparates. Chirurg 77:943-962

15. Schmidt HGK, Gerlach UJ, Wurm M, Hadler D (2004) Offene Gelenkrevision, Desaster-Management. In: Hendrich C, Frommelt L, Eulert J (Hrsg) Septische Knochen- und Gelenkchirurgie. Springer, Berlin Heidelberg New York

16. Schnettler R, Steinau HU (2004) Septische Knochenchirurgie. Thieme, Stuttgart New York

17. Tiemann AH, Hofmann GO (2008) Die akute postoperative Osteitis: Therapeutische Strategie zu einer oft verkannten Entität. CHAZ 9:371-377

18. Tiemann AH, Braunschweig R, Hofmann GO (2008) Kontiuitätserhalt oder Segmentresektion. Entscheidungshilfen bei der Sanierung der Osteitis am Schaft langer Röhrenknochen. Trauma Berufskrankh [Suppl 2] 11:179-183 